\title{
TITAN-XIII: sprawling-type quadruped robot with ability of fast and energy-efficient walking
}

\author{
Satoshi Kitano ${ }^{1 *+} \mathbb{0}$, Shigeo Hirose ${ }^{2 \dagger}$, Atsushi Horigome ${ }^{1 \dagger}$ and Gen Endo ${ }^{1 \dagger}$
}

\begin{abstract}
In this paper, we discuss development of a sprawling-type quadruped robot named TITAN-XIII which is capable of high speed and energy efficient walking. We consider a sprawling-type quadruped robot is practical, because of its high stability which comes from the large supporting leg polygon and the low center of gravity. However in previous researches, the speed and the energy efficiency of a sprawling-type quadruped robot is lower than a mammal-type quadruped robot. Since cost of transport (COT) can be reduced by increase of walking velocity, we decided to design a fast walking sprawling-type quadruped robot. As a demonstrator, we developed the sprawling-type quadruped robot named TITAN-XIII. For a lightweight and compact leg, the right-angle type wire driven mechanism is adopted to the robot. To confirm its performance, several experiments were carried out and the robot walked at $1.38 \mathrm{~m} / \mathrm{s}$ and COT of 1.76 was achieved. Finally, we compared the performance of TITAN-XIII with other quadruped robots, and confirm that its performance is almost same level as mammal-type quadruped robots.
\end{abstract}

Keywords: Quadruped robot, Wire driven, Dynamic walking

\section{Background}

There has been increasing interest in walking robots which can walk over uneven terrain where it is difficult for wheeled or crawler type robots to travel. Among the various types of walking robots, the four-legged walking robot has the minimum number of legs establishing static walking, and thus is regarded as highly practical configuration.

Quadruped robots can be classified into two groups, a mammal-type and a sprawling-type, according to its leg configuration (Fig. 1). Here, a mammal-type means the robot which locates its foot vertically downward from the base of the leg as a standard posture (Fig. 1a). A sprawling-type means the robot whose first leg segment (thigh) is in horizontal direction and second leg segment (shank) is in vertical direction as a standard posture (Fig. 1b).

\footnotetext{
*Correspondence: kitano.s.ac@m.titech.ac.jp

${ }^{\dagger}$ All authors contributed equally.

${ }^{1}$ Department of Mechanical and Aerospace Engineering, Tokyo Institute of Technology, Meguro-ku, Ookayama 2-12-1, 152-8550 Tokyo, Japan
} Full list of author information is available at the end of the article
There are several advantages in a mammal-type quadruped robot. First, a mammal-type quadruped robot can walk faster than a sprawling-type quadruped robot by utilizing two actuators (e.g., hip and knee) in each leg. Second, a mammal-type quadruped robot required small torque on each joint by straighting its leg, especially when the robot stands. Third, because of the its small footprint, the robot can walk through narrow space or side of a cliff like a mountain goat doing.

Recently, as a represented by BigDog [1] developed by BostonDynamics, mammal-type quadruped robots is studied actively. HyQ [2] which is a hydraulic quadruped robot, is capable to walk over rough terrain using the active compliance of the joints. MIT Cheetah [3] which is an electric quadruped robot, achieves energy efficient walking and dynamic jumping by developing a high torque density electromagnetic motor. StarIETH [4] which equips the series elastic actuator (SEA) on each joint, can walk on rough terrain interacting with ground dynamically. CheetahCub [5] and Tekken [6] also equip springs in its leg structure and utilize the springs for walking on rough terrain. Additionally,

\section{Springer}




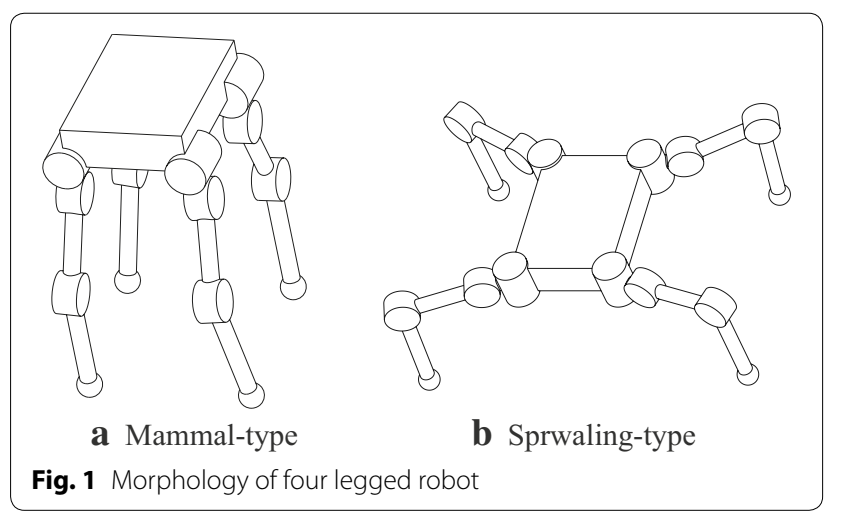

in Learning Locomotion Program held by Defense Advanced Research Projects Agency (DARPA), LittleDog is developed by BostonDynamics and several US universities studied about learning and planning of locomotion with the developed robot [7-9].

On the other hand a sprawling-type quadruped robot has another features. First, the robot has high stability, because the robot can locate its center of gravity at low position and have a wider supporting leg polygon. Second, the robot has wide range of motion because of its proximal yaw axis, therefore it can choose foot placement widely. Third, since its center of gravity is low, even if the robot falls down, the damage to the robot is considered to be relatively small. Additionally, it is easy to use the body of the robot as a fifth foot depending on the terrain.

Considering about a practical legged robot, the robot have to be capable of carrying objects or doing some operation rather than just moving. Otherwise, just for moving over rough terrain, another type of robot such as a flying robot also can perform. To carry objects, lowering center of gravity reduces the risk of a falling and damages. For doing some operation, wide range of motion helps to establish a stable platform like an outrigger. Thus, we consider that a sprawling-type quadruped robot is a practical quadruped robot.

So far, various types of sprawling-type quadruped robot has been developed and their high rough terrain adaptability is reported. TITAN III [10] walked over stair case autonomously using "the whisker sensor" equipped on each foot. SILO4 [11] has a compact leg using the spiroid gear, and walk over a step and a slope using a force sensor on each foot. MRWALLSPECT IV [12] succeeded to walk over various type of rough terrain autonomously by introducing the body workspace concept. Compact rescure robot (CRR) [13] achieves big payload more than its weight by using a pneumatic actuator on each joint.

Although a sprawling-type robot is highly practical, it is generally said that a sprawling-type quadruped robot has following disadvantages. First, because of the sprawling first segment, the proximal pitch axis always has to generate the torque to support its own weight, therefore its energy efficiency seems to be low. Second, mainly the walking velocity is generated by only proximal yaw actuator, its walking speed would be limited compared to mammal-type. Although the walking velocity and the energy efficiency are very important parameter for a moving machine, so far there is almost no research about walking velocity and energy efficiency of a sprawling-type quadruped robot.

Thus, the purpose of this research is to investigate and improve walking velocity and energy efficiency of a sprawling-type quadruped robot. Contribution of this paper is proving that a sprawling-type quadruped robot can walk fast and efficiently against common belief of the sprawling-type quadruped robot, and proposing a mechanical design of a sprawling-type quadruped robot to achieve fast walking and energy efficiency.

This paper first discuss how to improve walking velocity and energy efficiency. Then, we explain in detail about the sprawling-type quadruped robot named TITAN-XIII which developed as a demonstrator. Finally, we carried out experiments to verify its walking velocity and energy efficiency, and compare its performance with other quadruped robots.

\section{Design concept}

In this section, we discuss how to improve walking speed and energy efficiency of the sprawling-type quadruped robot.

\section{Walking velocity and energy efficiency}

First of all, walking velocity and energy efficiency are not independent parameter, and there is a strong relationship between two parameters. As a criterion of walking efficiency of a legged robot, cost of transport (COT), also known as specific resistance [14] is used broadly. COT is defined as follows:

$$
C O T=\frac{E}{m g d}=\frac{P}{m g v},
$$

where $E$ : consumed energy [J], $m$ : mass of the robot $[\mathrm{kg}]$, $g$ : gravitational acceleration $\left[\mathrm{m} / \mathrm{s}^{2}\right], d$ : moving distance $[\mathrm{m}], P$ : consumed power $[\mathrm{W}]$, $v$ : walking velocity $[\mathrm{m} / \mathrm{s}]$. Smaller COT means higher energy efficiency. The equation shows that increase of velocity causes decreasing COT. However, normally increase of velocity also causes increase of the mechanical energy which is required to move forward.

In case of robots, generally consumed energy means all of the energy consumption of the robot, including electric circuits such as microcontrollers and sensor devices which does not directly contribute to generate 
mechanical work. Additionally in case of legged robots, the robot have to support gravitational force which wheeled or tracked type robot does not need to support. These energy consumption is not small in reality. For example, in case of the sprawling-type quadruped robot TITAN-VIII [15], the electric energy consumption is almost $40 \%$ of its total energy consumption when the robot walks at $0.1 \mathrm{~m} / \mathrm{s}$ [16]. However, unlike the mechanical energy, these consumed energies are proportional to a running time. Therefore by reducing a running time, e.g. increase of walking velocity, total COT can be reduced because of decrease of the electric energy and the gravitational support energy.

\section{Increase of walking velocity}

To increase the walking velocity, the foot velocity in the moving direction should be as fast as possible. In case of a legged robot, the leg is always reciprocated. Hence, the inertia of the leg should be small as much as possible to achieve fast leg movement and high energy efficiency.

In addition to the foot velocity, walking gait is also important for increasing walking velocity. In the previous researches of sprawling-type quadruped robots, the crawl gait is mainly focused [12,15], because of its static stability. However the walking speed of the crawl gait is relatively slower than other dynamic walking gaits. On the other hand, in researches of mammal-type quadruped robots, the trot gait is mainly used.

To compare each gait, we estimate the walking velocity of legged robot which can be defined as follows:

$$
V=\frac{(1-\beta) U}{\beta},
$$

where $U$ : a foot velocity, $\beta$ : walking duty factor $(\beta>0.5)$. According to the equation, the maximum walking speed of the crawl gait is $\frac{1}{3} U$ at minimum duty factor of 0.75 . Similarly, the maximum walking speed of the trot gait is $U$ at minimum duty factor of 0.5 . The trot gait is theoretically three times higher than the crawl gait. Thus, it is very effective to choose the low duty factor gait such as the trot gait to increase walking velocity.

Additionally, we consider the trot gait is also having high stability on rough terrain. Assuming the robot walking with the trot gait and it starts to fall because of unexpected disturbances or errors, the robot rotates around diagonal supporting line. However in both rotating directions, the swinging legs exist, and one of the swinging legs hits the ground and forms a supporting leg polygon with the supporting legs. Because of the newly formed supporting polygon, the robot will maintain standing posture if the tumbling velocity is low.

Thus, to achieve fast walking on a rough terrain, we believe the trot gait is most suitable.

\section{Reducing gravitational support energy}

Although the electric and the gravitational support component of COT can be decreased by increase of velocity, still the electric energy consumption and gravitational support energy consumption remain in the system. Especially, in case of the sprawling-type quadruped robot, gravitational support energy tend to be bigger than the mammal-type quadruped robot, because of its horizontally extended leg. To decrease gravitational support energy, it is considered to use non-backdrive mechanism such as a worm gear and a screw mechanism. However this kind of mechanisms has disadvantages which is heaviness and low transmission efficiency. Because of these disadvantages of non-backdrive mechanisms, it does not suit for a fast walking quadruped robot. As a practical solution, the reduction ratio of an actuator supporting gravitational power should be as high as possible, within achieving required velocity for walking motion.

Summarize above discussion, the important points to achieve high speed and energy efficiency are below.

- COT can be reduced by increasing walking velocity.

- By using a low duty factor gait such as the trot gait, the walking velocity can be increased.

- To reduce gravitational support energy, the actuator which supports gravitational force should have large reduction ratio.

\section{Hardware design}

As a demonstrator model, we developed the new sprawling-type quadruped robot named TITAN-XIII in accordance with the previous design concept. Overall view of the robot is shown in Fig. 2 and Table 1 shows the specification of the robot. We also define the standard posture as shown in Fig. 3 and the size of Table 1 is based on this standard posture.

The weight, reduced by decreasing the component count as far as possible and using carbon fiber reinforced plastic for main structure of each leg segment, is only $5.65 \mathrm{~kg}$ including the battery. As an actuator, customized DC brushless motors (FX1206-11 made by Nippo Denki Co., Ltd., max. power $68 \mathrm{~W}$ ) is used for all of the joint. As a result, the power-weight ratio which is summation of maximum output of actuators divided by weight of the robot is $144.4 \mathrm{~W} / \mathrm{kg}$, which is about 4 times higher than the previously developed sprawling-type quadruped robot TITAN-VIII [15] with similar dimension whose power-weight ratio is $40 \mathrm{~W} / \mathrm{kg}$.

Additionally a modularized design was adopted for maintainability, and the robot is composed of four identical leg unit. This makes it possible to easily maintain each unit, and if a malfunction occurs, function can be restored simply by replacing the malfunctioning unit. 


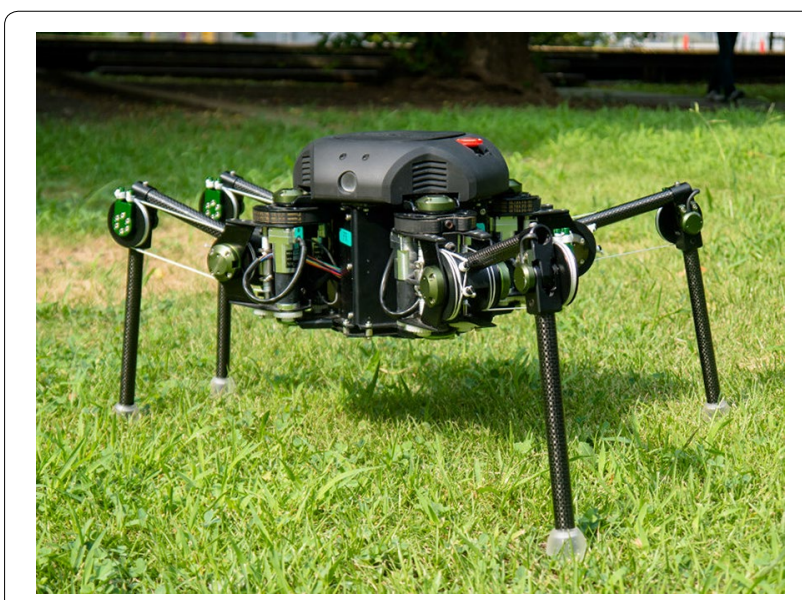

Fig. 2 Sprawling-type quadruped robot TITAN-XIII

With regard to the power source, power is supplied to the actuators and microcontrollers by an LiFePO4 battery (26.4 V, $1100 \mathrm{mAh}$ ) made by A123 and installed in the center of the body.

\section{Mechanism of leg unit}

Figure 4 shows the overall view of the leg unit and Fig. 5 shows a schematic and detailed diagram of the leg unit. Table 2 shows the specification of the leg unit.
Table 1 Specification of the TITAN-XIII

\begin{tabular}{ll}
\hline Characteristic & Value \\
\hline Size $(\mathrm{L} \times \mathrm{W} \times \mathrm{H})$ & $213.4 \times 558.4 \times 340.0 \mathrm{~mm}$ \\
Weight $($ W/o battery) & $5.29 \mathrm{~kg}$ \\
Weight (with battery) & $5.65 \mathrm{~kg}$ \\
Payload & $5.0 \mathrm{~kg}$ \\
Battery & LiFe $26.4 \mathrm{~V} 1100 \mathrm{mAh}$ \\
Battery run time & approx. $20 \mathrm{~min}$. \\
\hline
\end{tabular}

The leg unit has three degrees of freedom and consists of a planar mechanism with two degrees of freedom employing two pitch axes (Axis 2 and Axis 3), and a yaw axis (Axis 1) which rotates the planar mechanism. To reduce the inertia of the leg around the yaw axis, the actuators for pitch axes are placed close to the yaw axis and transmit power to each joint by using wires.

Two wires each are used to drive one joints. Since the diameter of idler pulley on Axis 2 and the diameter of the pulley fixed on Axis 3 are the same, this planar mechanism comprises a parallel mechanism.

For the transmission of the yaw axis (Axis 1), a timing belt and a pulley are used. Although a pulley is made with metal material usually, but this time the pulley made with poly-acetal plastic is used as an output pulley for lightweight. Since it is difficult to have small teeth on a plastic
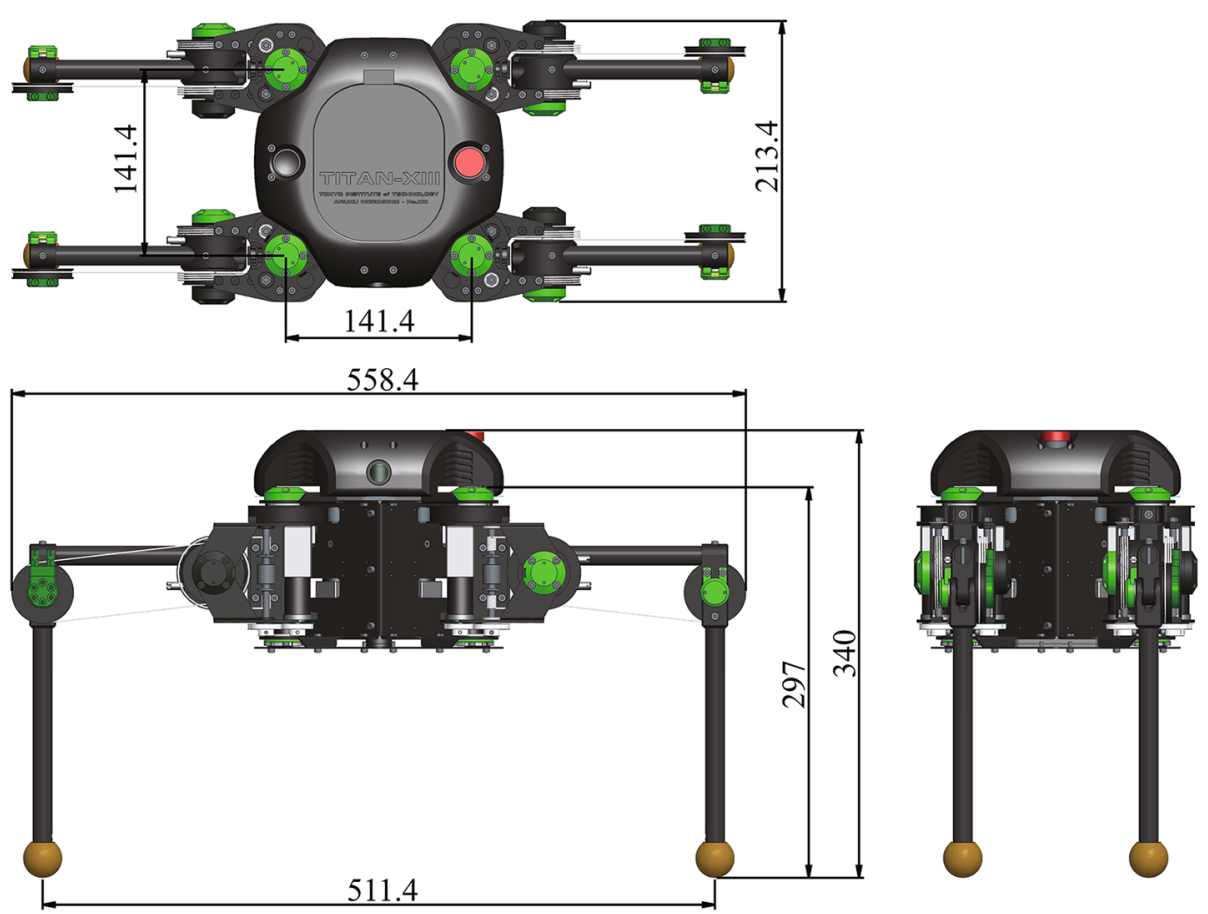

Fig. 3 Trihedral figure of TITAN-XIII in standard posture 


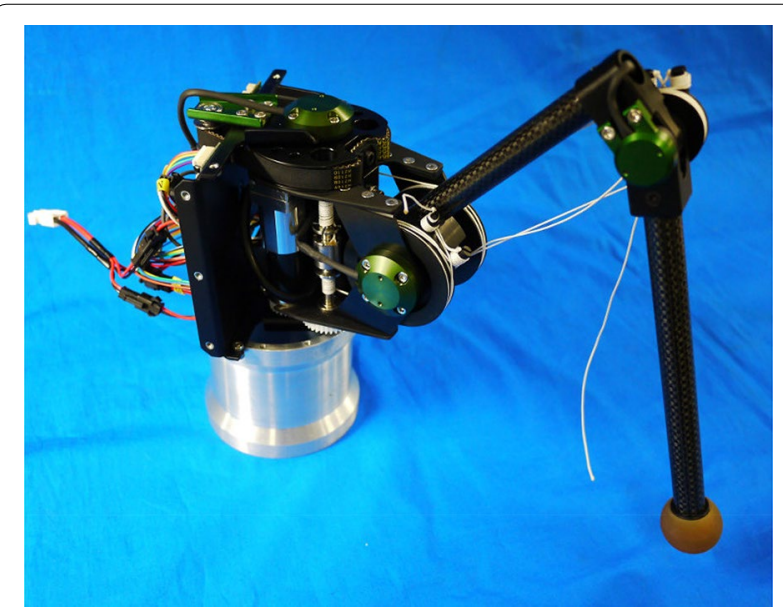

Fig. 4 Leg unit of TITAN-XIII

pulley, the pulley has a dent to fasten a timing belt with a screw via tensioner plate as show in Fig. 6.

The screw fixing the timing belt to the pulley also work as tensioner which is usually achieved by additional idler pulley. This pulley with dent can not be used in case of requiring rotation of $360^{\circ}$ or more, but can be regarded as effective at case of where only $180^{\circ}$ of range of motion is required, such as the Axis 1. As shown in Fig. 7, the motor is fixed to the back side of the leg base section, and the timing belt is connected to the output axis via an opening provided in the leg base.

The gear reduction ratio of each axis is shown in Table 2. As we discussed above, the reduction ratio of Axis 1 is set to low to achieve high walking speed. The reduction ratio of Axis 2 is set to high, as the leg can support whole weight of the robot. In case of Axis 3, we consider it does not require the so much torque as Axis 2 .

Therefore Axis 3 uses a smaller pulley and lower gear reduction ratio than Axis 2.

The range of motion of the planer mechanism is shown in Fig. 8. All of the joint have range of motion of $\pm 90^{\circ}$, and makes it possible to completely fold up a leg to the inside.

\section{Wire driven mechanism}

As we mentioned above, the wire driven mechanism is used for the Axis 2 and the Axis 3. Figure 9 shows the wire driven mechanism for the Axis 2. For the Axis 3, almost symmetric design is used with extra relay pulley to transmit the Axis 3.

There are several reasons, aside from transmitting power to a remote location, why a wire driven mechanism

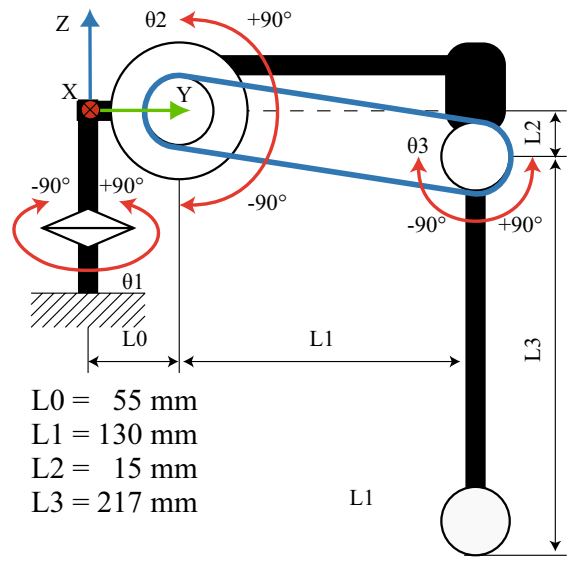

a Schematic diagram
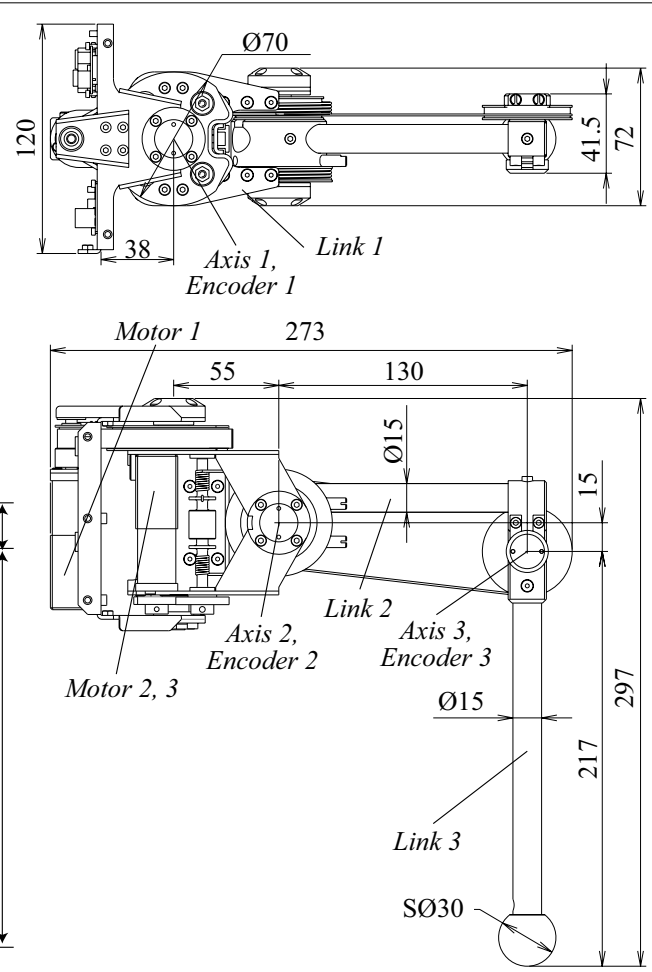

b Detailed diagram

Fig. 5 Leg Mechanism of TITAN-XIII. a The basic structure of the leg unit and range of motion of each joint. $\mathbf{b}$ The detailed drawing of the leg unit and indicates the position of the motors and the encoders 
Table 2 Specification of the leg unit

\begin{tabular}{|c|c|}
\hline Weight & $1.2 \mathrm{~kg}$ \\
\hline Actator & $\begin{array}{l}\text { Nippo Denki: FX1206-011 brushless } \\
\text { DC motor (max. output:68W) }\end{array}$ \\
\hline Motor driver & Hibot: 1BLDC power module \\
\hline Reduction mechanism for the axis1 & Planetary gear, timing belt \\
\hline $\begin{array}{l}\text { Reduction mechanism for the axis2, } \\
3\end{array}$ & $\begin{array}{l}\text { Planetary gear, spur gear, wire and } \\
\text { pulley }\end{array}$ \\
\hline \multicolumn{2}{|c|}{ Reduction ratio (each reduction ratio Motor1: $115(23.04,5)$} \\
\hline of mechanism) & Motor2: $273(23.04,1.4,8.5)$ \\
\hline & Motor3: $163(23.04,1,7.1)$ \\
\hline Sensor & $\begin{array}{l}\text { Sastinable robotics: } 16 \text { bit absolute } \\
\text { magnet encoder }\end{array}$ \\
\hline Power weight ratio & $170 \mathrm{~W} / \mathrm{kg}$ \\
\hline
\end{tabular}

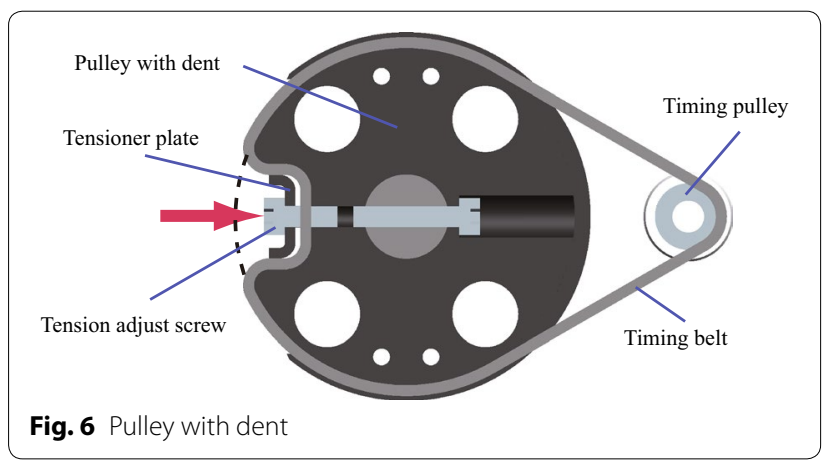

is used instead of usual gears for the final output stage of the joint mechanism.

The most important feature is that the wire driven mechanism can achieve higher allowable torque density (allowable torque of reduction mechanism $[\mathrm{Nm}]$ / weight of reduction mechanism $[\mathrm{kg}]$ ) comparing to other reduction mechanisms. In case of a gear reduction mechanism, the power can be reliably transmitted. However the output torque is supported by one or two of the gear teeth, and in order to output high torque, the module of the gear must be large or material of the gear need to have high yield strength which generally makes a mechanism heavy such as steel. The same is true in the case of a timing belt, which can transmit power to a separate axis just like wire. In the case of the wire driven mechanism, strength of wire that transmits torque between pulleys, limits allowable torque. On the side of pulley in which wire wound, since wire tension is attenuated along with winding onto the pulley surface, even plastic material can endure wire tension.

Additionally the wire driven mechanism does not have backlash which is major problem in gear reduction mechanism.
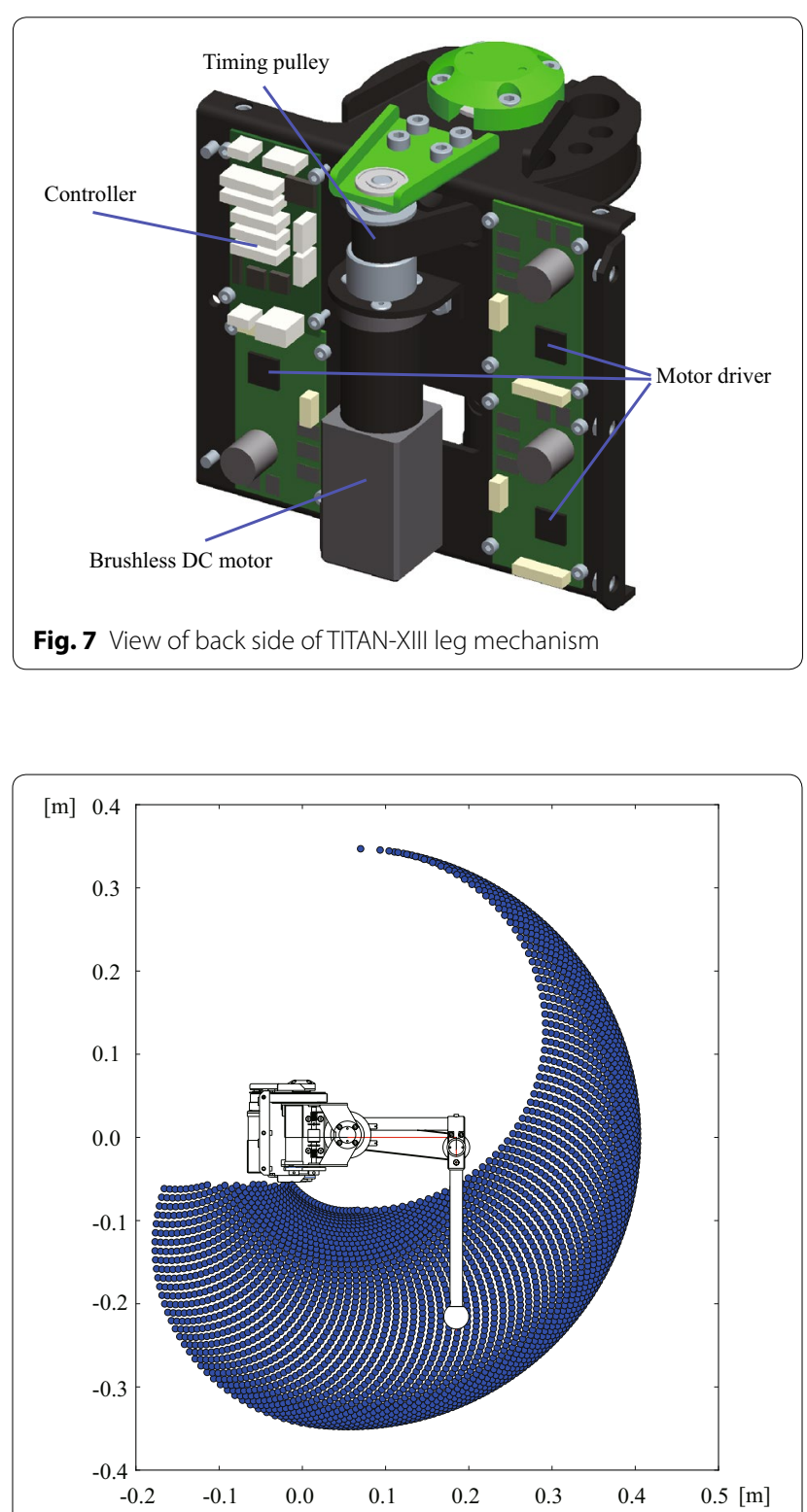

Fig. 8 Range of motion of planer mechanism. The plot is the calculated by forward kinematic inputting angle of Axis 2 and Axis 3. Input angle are changed from $-\pi / 2 \mathrm{rad}$ to $\pi / 2 \mathrm{rad}$ by $0.05 \mathrm{rad}$

In case of normal gear reduction mechanism, increasing reduction ratio only affects pitch diameter ratio between an input gear and an output gear. However in case of wire reduction mechanism, increasing reduction ratio also causes increasing number of windings of wire and axial direction length of the pulley if range of motion of output pulley is kept.

As shown in Fig. 10a, in most of cases axes of the output pulley and the input pulley are arranged in parallel. In this configuration the whole mechanism needs to expand 


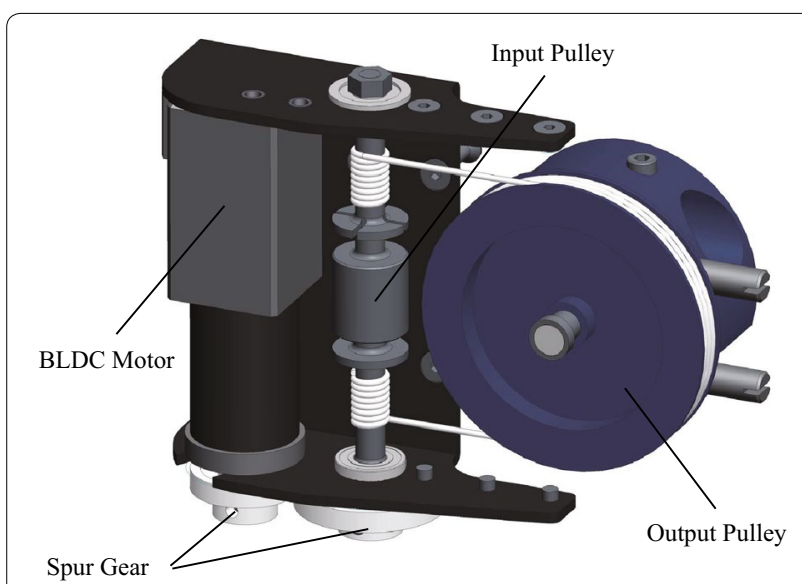

Fig. 9 Wire driven mechanism which is comprised of a brushless DC geared motor, spur gears, two wires, and input and output pulleys

in both direction, radial direction of the output pulley and axial direction of the input pulley, as the reduction ratio is increased. Additionally, when the input pulley is rotated, the point where the wire separates from the input pulley moves in the axial direction, and thus the output pulley must have equivalent length in the axial direction.

Therefore we choose another pulley arrangement, where the input pulley and output pulley are arranged at right angles as shown in Fig. 10b. In this configuration, the axial direction length of the input pulley, which increases as the reduction ratio increases, is matched with the radial direction of the output pulley, and this conserves space.

A space-saving new wire reduction mechanism achieved a high reduction ratio of 8.46 on Axis 2, taking into account an input pulley diameter of $5.5 \mathrm{~mm}$, output pulley diameter of $54.5 \mathrm{~mm}$, and wire diameter of $1.05 \mathrm{~mm}$. In the case of Axis 3 output pulley diameter is $45 \mathrm{~mm}$ and the reduction ratio of 7.1 is achieved.

One problem of this wire driven mechanism with rightangle type is that, as the output pulley rotates the point of separation of the wire on the input pulley moves up and down because of the winding of the wire as shown in Fig. 11. This motion changes the path length of the wire and adds extra tension on the wire. Theoretically speaking in order to solve this problem, an input pulley or an output pulley must have sliding degree of freedom along its axis to absorb the up and down motion, but this makes the mechanism large and complicated. Therefore, we examined the elongation of the wire in right-angle type wired driven mechanism. In Fig. 11, $L$ is the length of the wire between the input pulley and the output pulley in the standard posture where theoretically the wire is perpendicular to the input pulley. Now assuming the output pulley rotates $\theta$, and the input pulley rotates $\phi=$ reduction ratio $\times \theta$. Then the point of separation of the wire is moved distance $d l=d \times \phi / 2 \pi$, where $d$ is diameter of the wire. Along with the moving of the point of separation of the wire, the length of wire between the input pulley and the output pulley is changed to $L^{\prime}$. This new wire length $L^{\prime}$ can be expressed as $\sqrt{d l^{2}+L^{2}}$. As a result, wire elongation $L^{\prime}-L$ can be calculated. Since Axis 2 has larger reduction ratio of 8.46 than Axis 3 we will check elongation of Axis 2. Considering $L$ is $40 \mathrm{~mm}$ and output pulley rotates $\pm 90^{\circ}$, estimated elongation $L^{\prime}-L$ is only $0.06 \mathrm{~mm}$. Thus we consider this elongation is sufficiently tolerable and does not need a complicated mechanism.

\section{Synthetic fiber rope}

For the wire driven mechanism, the property of the wire affects its performance. Conventionally a stainless steel wire rope is commonly used for the wire driven mechanisms including TITAN-VIII [15]. However, it is difficult to wind stainless steel wire onto a pulley which has an extremely small diameter such as the input pulley used for the TITAN-XIII. Thus, we decided to use a synthetic fiber rope due to its expected superior flexibility to stainless steel wire. There are many types of synthetic fiber rope has been developed. Among them, we choose Ultra-high-molecular-weight polyethylene (UHMWPE) a.k.a Dyneema, because of its high tensile strength of 2.6 GPa [17] which is almost five times higher than SUS304 (around 0.5MPa). As a Dyneema rope, we used DB28HSL (developed by Hayami Indutstry Co., Ltd, shown in Fig. 12) which is braided of eight Dyneema strands. Table 3 shows the basic properties of the Dyneema rope and stainless steel wire rope. Because of braiding, breaking force of the DB-28HSL is low considering tensile strength of the material, but it is still higher than stainless steel wire rope. Thus, we consider Dyneema is suitable for robotic application.

The problem of using the wire driven mechanism is an elongation of a wire, which decreases mechanical bandwidth. Therefore to be able to adjust wire tension easily a co-axial tensioner shaft shown in Fig. 13 was devised as the input pulley. The co-axial tensioner shaft has a dual structure made up of a core shaft which passes through the center of the input axis pulley, and an outer shaft which covers the lower half. Each shaft is connected with a compact roller type one-way clutch (NTN: HF0612, torque capacity $1.76 \mathrm{Nm}$ ). Due to the one-way clutch, the two shafts turn relative to each other only in the direction which increases wire tension, and thus it is possible 


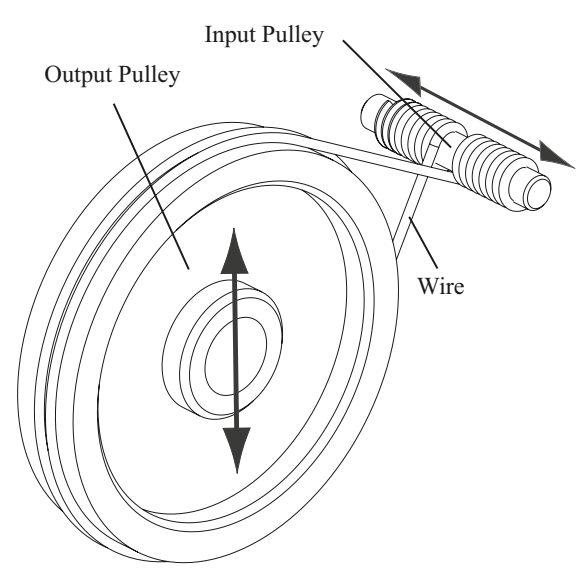

a Parallel type

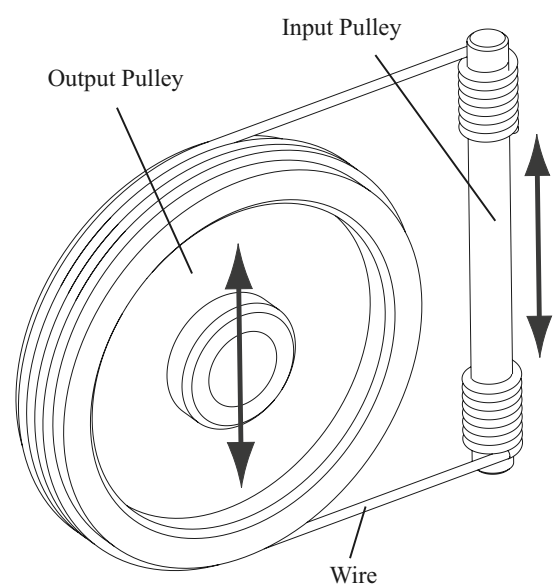

b Right angle type

Fig. 10 Comparison of the pulley arrangement of wire reduction mechanism. The arrows show the expanding direction of each pulley if reduction ratio is increased. In case of $\mathbf{a}$ expanding direction is different, but in case of $\mathbf{b}$ expanding direction is same

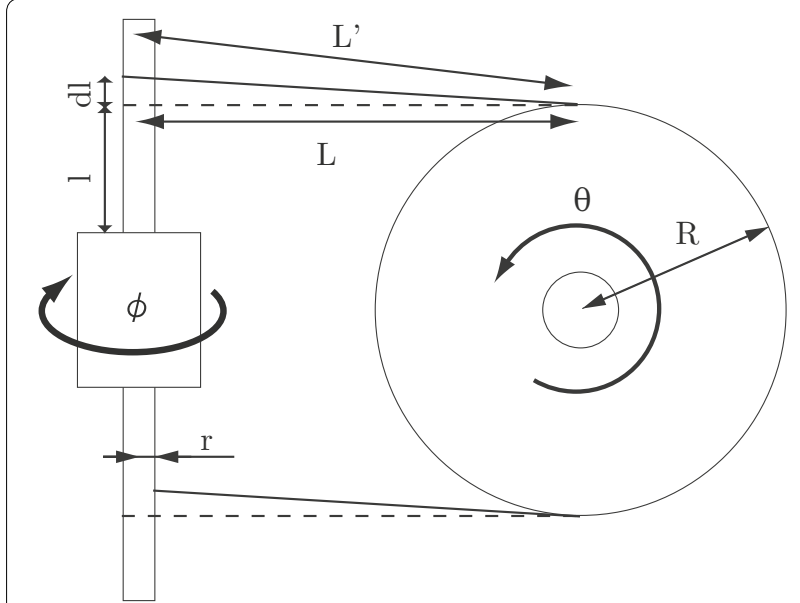

Fig. 11 Wire elongation due to pulley rotation

to adjust tension of the wire by rotating one of the shafts. The wire which is wound to the core shaft transmit power directly to the motor, but the wire wound to the outer shaft transmit power via one-way clutch.

Since the one-way clutch has maximum transmittable torque, the transmittable torque of the outer shaft is limited by torque capacity of the one-way clutch. However walking robots normally require high torque in one direction that gravity works, we deploy wire driven mechanism as the core shaft supports the direction of the gravity force.

\section{Control system}

Figure 14 shows an schematic of the control system of TITAN-XIII.
The main control program running on an external PC calculates each joint angle using inverse kinematics from an designed leg trajectory. This control program also provides interface to control a robot motion via a GUI and a joystick. Calculated joint angles are sent each $20 \mathrm{~ms}$ via WiFi communication using IEEE802.11ac (5 Ghz) avoiding radio frequency interference most commonly used $2.4 \mathrm{GHz}$ of bandwidth. The robot equips a small WiFi router and the TITech M4 controller (made by Hibot corp.) for communication with the router and the each leg unit.

Each leg unit equips the TITech SH2 Tiny Controller (made by Hibot corp.) and three motor drivers on the back of the leg unit as shown in Fig. 7. The microcontrollers receive joint angle data from the external PC and performs position control with a $1 \mathrm{kHz}$-cycle PID controller and interpolation of joint angle. Every joint angle is measured by the 16-bit magnet encoders made by Sustainable Robotics (using the AEAT-6600-T16 of AVAGO Technologies) mounted on each joint as an absolute angle sensor.

\section{Experiment}

\section{Position tracking of the foot}

Firstly, position tracking performance of the leg unit was tested. In the experiment, the leg unit executed walking motions in the air.

As a walking motion, the longitudinal acceleration trot gait which is described in detail in our previous work [18] was used. The commanded swing height and walking stride was kept at 0.05 and $0.18 \mathrm{~m}$. In the experiment, the robot was in the posture shown in Fig. 15 as a normal 


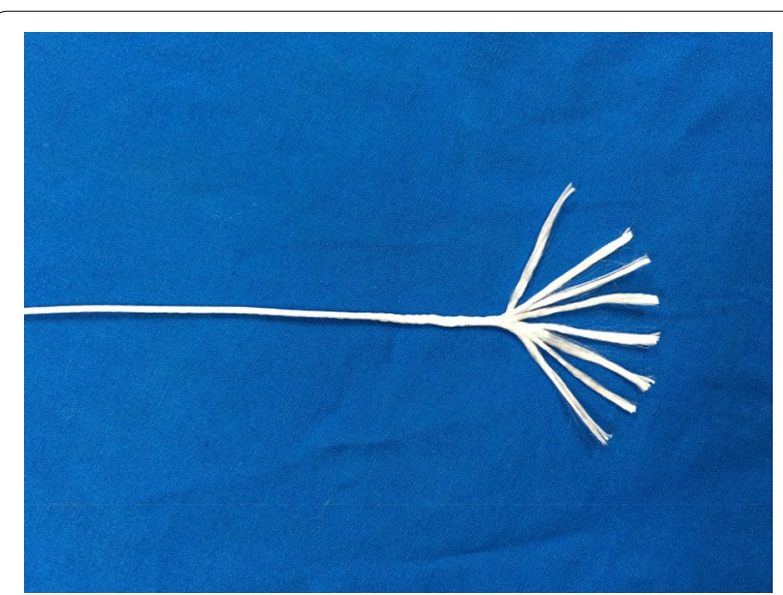

Fig. 12 Dyneema Rope DB-28HSL made by Hayami Industry Co., Ltd

Table 3 Properties of Dyneema rope and stainless steel wire rope

\begin{tabular}{lll}
\hline $\begin{array}{l}\text { Rope } \\
\text { material }\end{array}$ & $\begin{array}{l}\text { DB-28HSL } \\
\text { dyneema }\end{array}$ & $\begin{array}{l}\text { Stainless steel wire rope(1x7) } \\
\text { SUS304 }\end{array}$ \\
\hline Diameter & $1.05 \mathrm{~mm}$ & $1.0 \mathrm{~mm}$ \\
Breaking force & $1.38 \mathrm{kN}$ & $1.03 \mathrm{kN}$ \\
Weight per length & $0.66 \mathrm{~g} / \mathrm{m}$ & $5.24 \mathrm{~g} / \mathrm{m}$ \\
Specific strength & $2060 \mathrm{kNm} / \mathrm{kg}$ & $197 \mathrm{kNm} / \mathrm{kg}$ \\
\hline
\end{tabular}

Breaking force of DB-28HSL were measured by HAYAMI and weight per Length was measured by authors. Properties of stainless steel wire rope is refereed from JIS G3540(Wire ropes for mechanical control)

posture. The position of the foot was measured by using a motion capture system (Flex 13 by OptiTrack). Since we cannot put a reflection marker on the foot directly, we put the reflection marker on the Link 3 and calculated the position of the foot.

Figures 16 and 17 show the commanded and measured foot trajectory of walking motion at $0.33 \mathrm{~m} / \mathrm{s}$ of the target velocity $(0.94 \mathrm{~Hz})$ and $1.2 \mathrm{~m} / \mathrm{s}$ of the target velocity $(3.1 \mathrm{~Hz})$ respectively. In case of $0.33 \mathrm{~m} / \mathrm{s}$, the foot mostly follows the commanded trajectory. However, in case of $1.2 \mathrm{~m} / \mathrm{s}$ there is big errors especially while the transition between support phase and swing phase where right and left edge of the trajectory. The reason of these errors are considered as error of the magnetic encoder which is used for joint control. Since magnetic encoder has internal averaging function to output stable value, when the joint rotates fast, it outputs different value to actual joint angle position. Therefore, because of this magnetic encoder averaging issue, we were getting inaccurate feedback for the PID controller at high rotational speed. Then, when setting a commanded angle position to the joint, the output position exceeded the commanded position, resulting in a difference between the commanded foot position and the foot position measured by the motion capture system as shown in Fig. 17. However, since the measured stride exceeds commanded stride and its error to commanded stride is $11 \%$, we consider the robot can walk at target velocity or more.

\section{Walking experiment}

Walking experiments were conducted to confirm the walking velocity and the energy consumption of TITAN-XIII.

As a walking algorithm, the same longitudinal acceleration trot gait was used. In the experiment, the robot walked straight $3.0 \mathrm{~m}$ on a flat wooden plate. Through the experiments the duty factor was kept at 0.55 , but the target velocity and the stride which are control parameters are changed gradually. While the walking experiment, the normal posture of the robot was the posture shown in Fig. 15. Comparing to the standard posture (Fig. 15), the angle of the diagonal supporting line is increased to avoid collision between the forward feet and rear feet when the

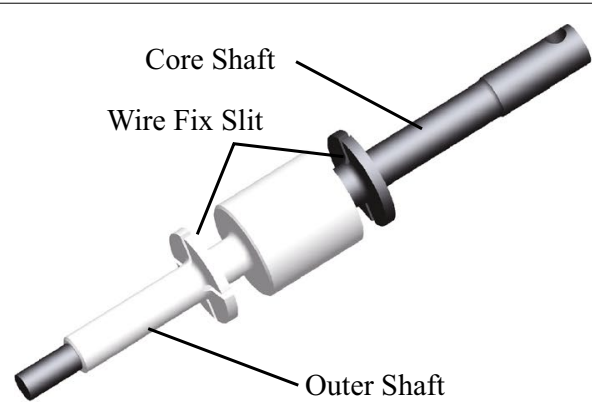

a Overview

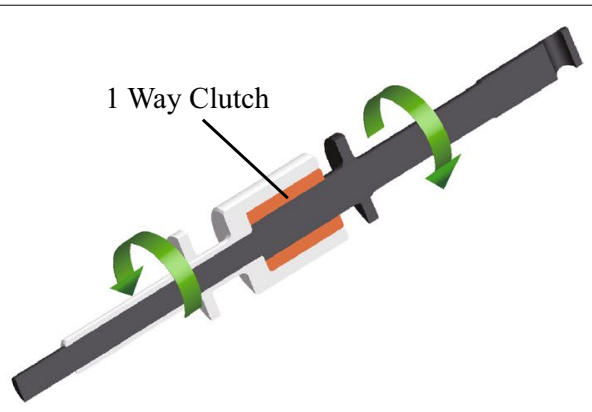

b Cross-section

Fig. 13 Co-axial tensioner shaft. a The overview of the co-axial tensioner shaft. The white part is the outer shaft and the gray part is the core shaft which penetrate the outer shaft. b The cross section of the co-axial tensioner shaft. The outer shaft and the core shaft are connected by one-way clutch and only rotate one direction which increases tension of the wire 


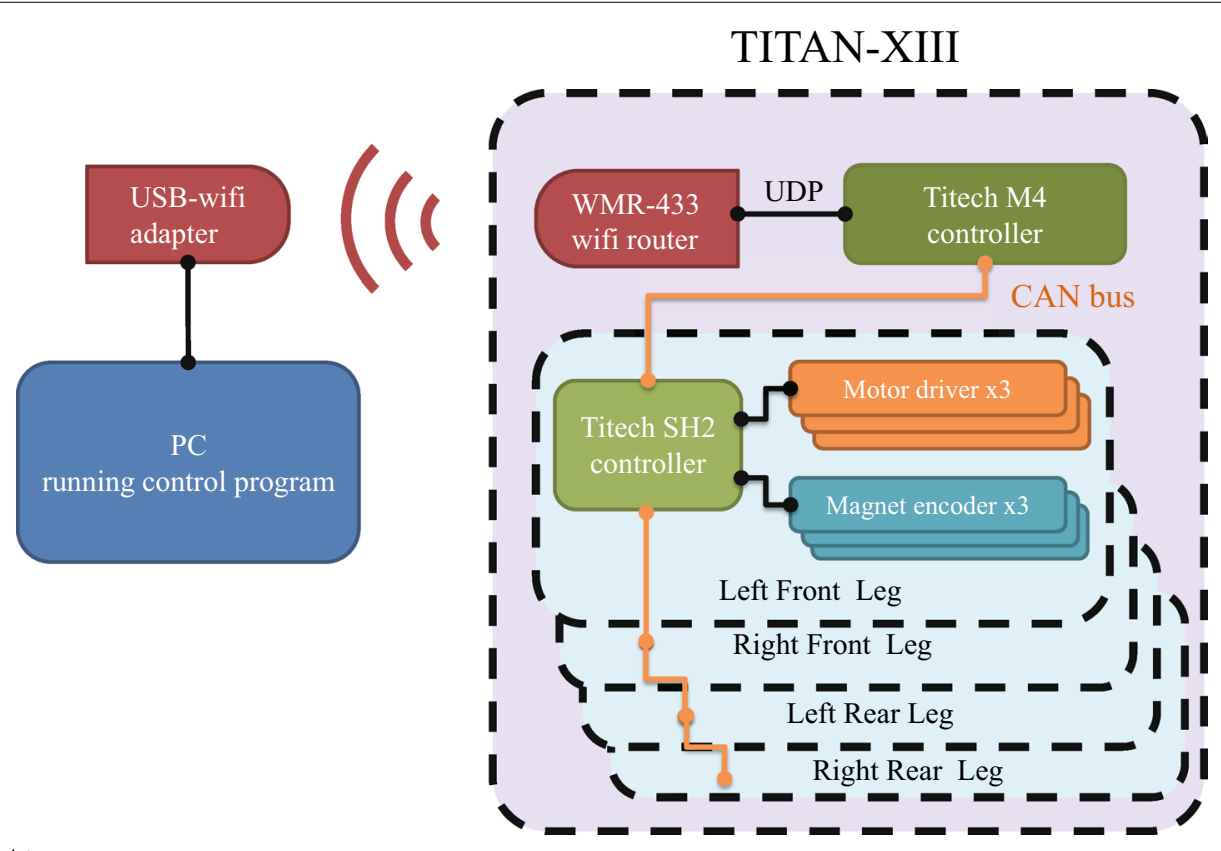

Fig. 14 System architecture

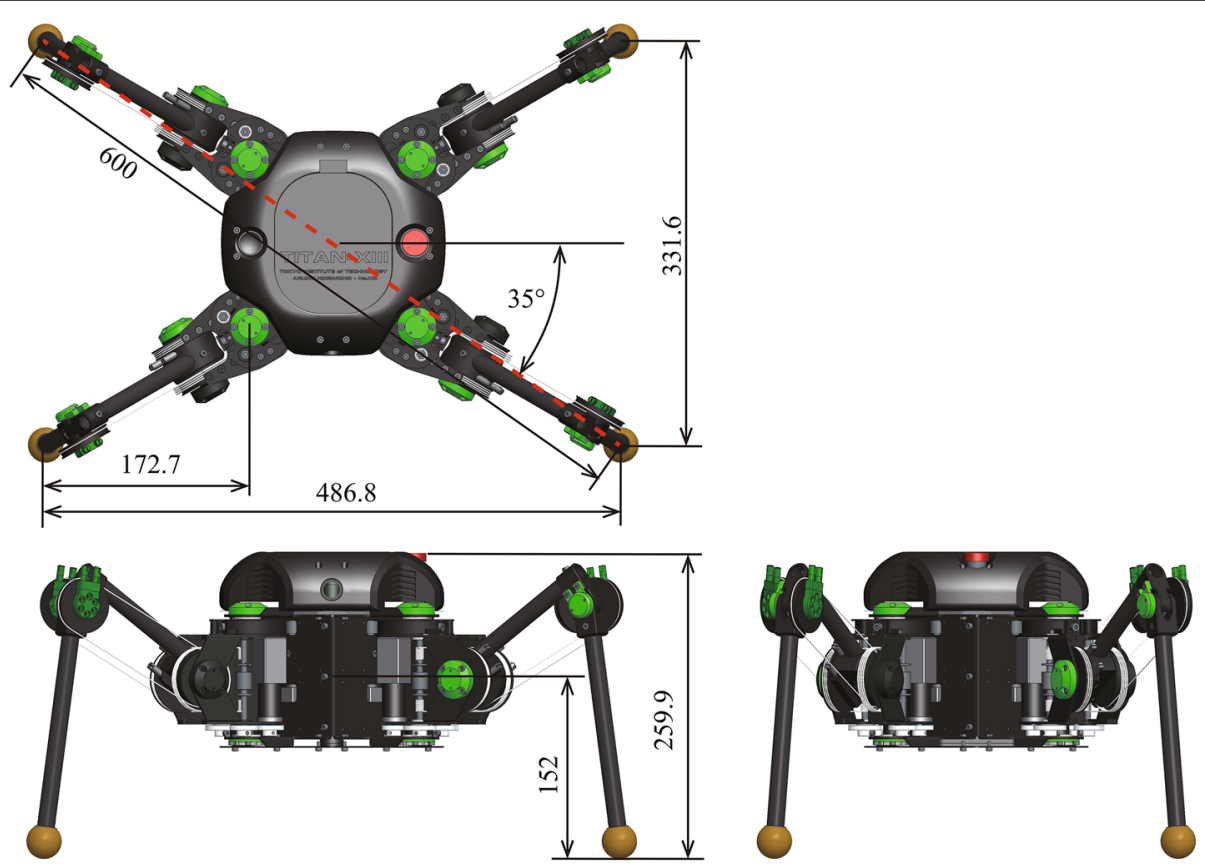

Fig. 15 Posture of the TITAN-XIII in the walking experiment. Red dot line shows the diagonal supporting line

stride is increased. Furthermore the height of the robot is decreased to avoid accidental complete tumbling. Relationship between a height of the center of gravity and stability of the legged robot is known as Normalized Energy Stability Margin (NESM) [19] which indicates a required energy to complete tumble normalized by the weight of a robot. According to the definition of NESM, lower center of gravity means higher stability.

Walking velocity is measured by using the motion capture system which also used in the position tracking of 


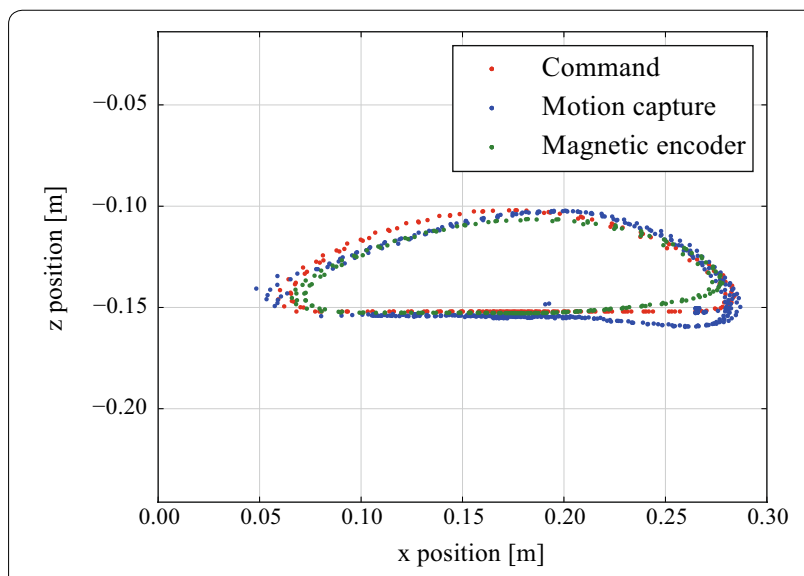

Fig. 16 Foot trajectory at target walking velocity $0.33 \mathrm{~m} / \mathrm{s}$ with $0.18 \mathrm{~m}$ of stride

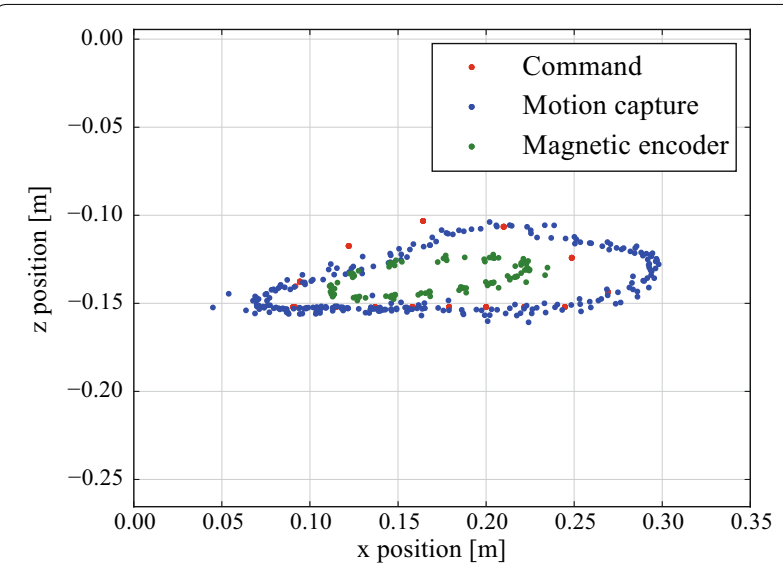

Fig. 17 Foot trajectory at target walking velocity $1.2 \mathrm{~m} / \mathrm{s}$ with $0.18 \mathrm{~m}$ of stride

the foot experiment. A power supply was connected to the robot to measure the consumed current although the battery is still installed in the robot. The consumed power was estimated by multiplying the $26.4 \mathrm{~V}$ of supply voltage and the measured current. This power includes not only an energy consumption in the actuators but also one in the electronics e.g. the microcontrollers, the WiFi router and a DC/DC converter. Each experiment was conducted three times with same parameter, and the result is shown as an average of three experiments.

Figure 18 shows side view of the sequence of walking experiment with $0.2 \mathrm{~m} / \mathrm{s}$ of the target velocity and $0.04 \mathrm{~m}$ of the stride and at this time the measured velocity was $0.22 \mathrm{~m} / \mathrm{s}$. As shown in the figure, the robot walked stably at constant speed and the body posture was kept in almost horizontal.

In the experiment, $1.38 \mathrm{~m} / \mathrm{s}$ of walking speed was achieved at target velocity $1.2 \mathrm{~m} / \mathrm{s}$ and at this speed the robot consumed $135 \mathrm{~W}$. The reason of higher walking velocity than target velocity is considered as longer stride which is also confirmed in Fig. 17. Figure 19 shows the commanded foot trajectory and measured foot trajectory while walking at $1.38 \mathrm{~m} / \mathrm{s}$. As shown in Fig. 19, actually the measured stride is larger than commanded stride.

We also conducted walking experiment with an additional mass. In static payload experiment, the robot can support $20 \mathrm{~kg}$ of a weight continuously by four legs. Assuming walking with trot gait, the robot have to support weight by only two leg in most of the sequence and can walk with additional mass of $10 \mathrm{~kg}$. However we set safety factor and conducted experiment with $5 \mathrm{~kg}$ of additional mass which is almost same as the weight of the robot.

The weight is fixed on the top of the robot putting the sponge rubber between the robot and the weight. Although the normal position of the foot is same as the Fig. 15, the height of the robot was decreased $0.02 \mathrm{~m}$ for stable walking. As same as previous experiment, the target velocity and the stride was gradually increased.

As a result, the robot successfully walked with additional mass and the fastest speed in the experiment was $0.9 \mathrm{~m} / \mathrm{s}$ with $0.15 \mathrm{~m}$ of stride at target velocity $1.0 \mathrm{~m} / \mathrm{s}$. At this speed the robot consumed $152 \mathrm{~W}$, whereas the robot consumed $103 \mathrm{~W}$ without payload at $0.96 \mathrm{~m} / \mathrm{s}$.

Increased power is considered to be consumed to support, accelerate and decelerate the additional weight.

\section{Energy efficiency}

By using the measured power and the velocity, COT of each walking parameter were calculated. The Fig. 20 shows the COT of the TITAN-XIII at each velocity and also the ratio of power consumption in COT. The shown velocity and COT is average of three trial.

As shown in the Fig. 20, the COT is decreased as the walking velocity increased. As a result, at $1.38 \mathrm{~m} / \mathrm{s}$ of the walking speed, the COT of 1.76 which is the lowest COT through the experiment was achieved.

To examine each component of power consumption, we define "the electric power consumption" which is the power consumption in the microcontrollers, the WiFi router and the DC/DC converter, "the gravitational support power consumption" as the power consumption of the robot standing still subtracted by the electric power consumption and "the mechanical power consumption" as the power consumption measured while walking subtracted by the electric power consumption and the gravitational support power consumption. In the experiment, electric power consumption is alway around $10 \mathrm{~W}$, and gravitational support power is around $13 \mathrm{~W}$ when the robot stands with four legs. 

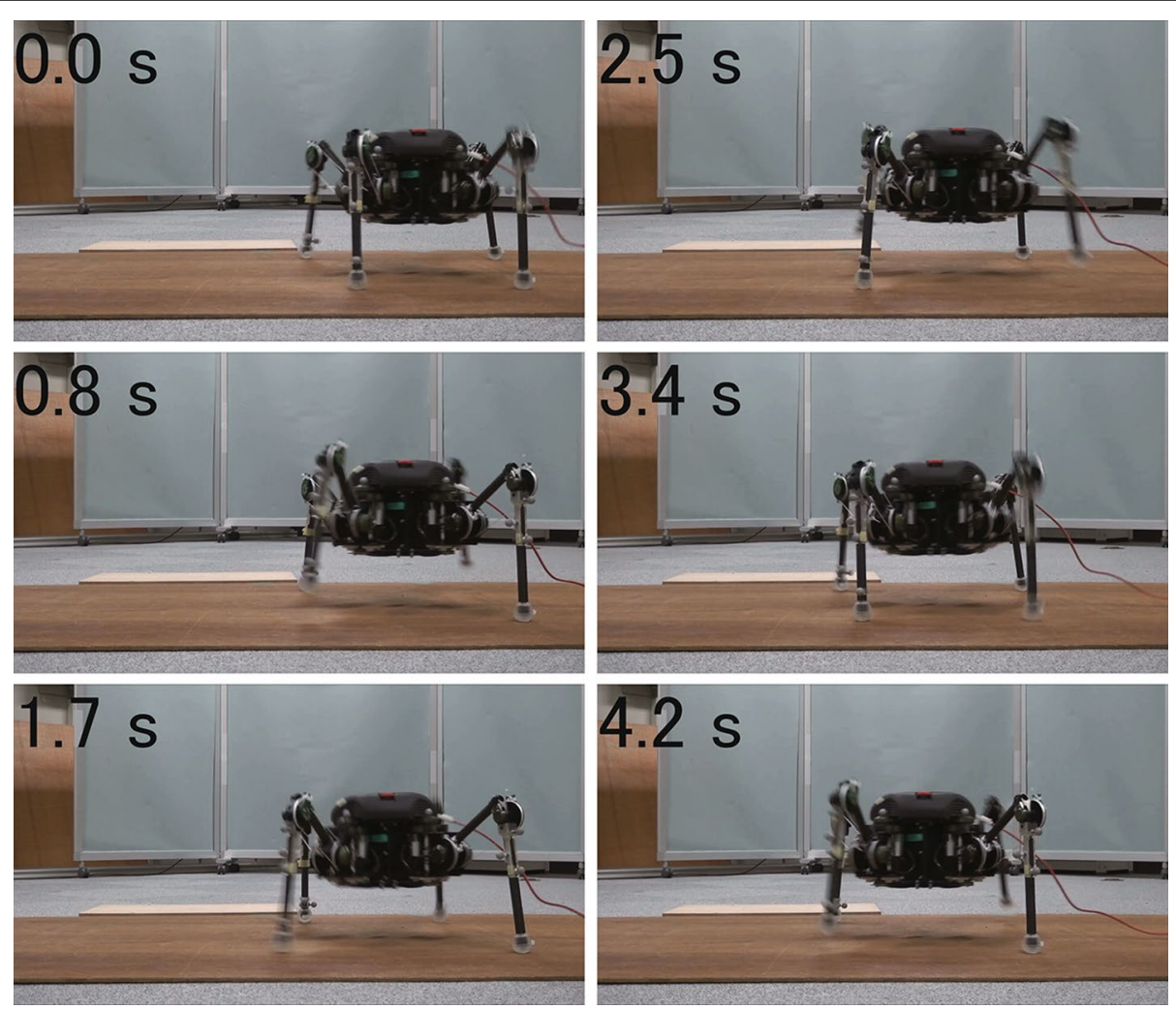

Fig. 18 Walking experiment at target velocity $0.2 \mathrm{~m} / \mathrm{s}$ with $0.04 \mathrm{~m}$ of stride. The measured velocity was $0.22 \mathrm{~m} / \mathrm{s}$

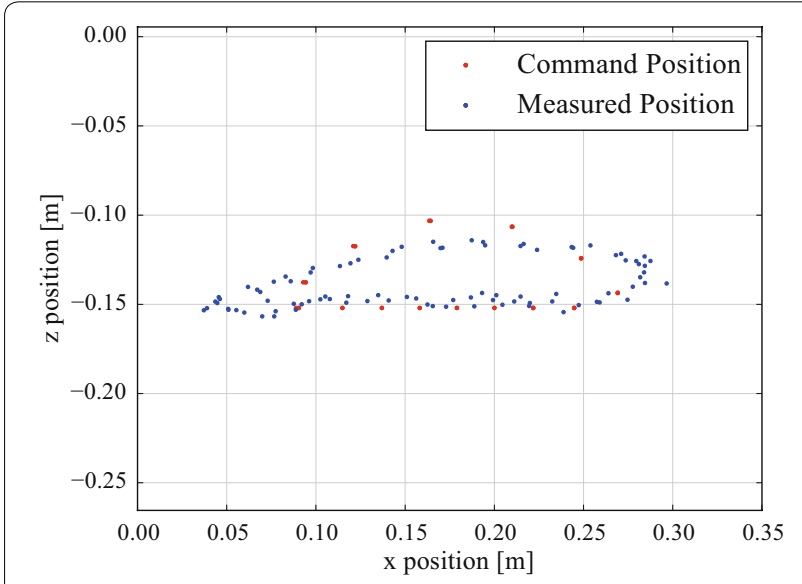

Fig. 19 Foot trajectory while walking on the ground at $1.38 \mathrm{~m} / \mathrm{s}$. Each foot trajectory is calculated with respect to the body coordinate system

The ratio of the electric power and the gravitational support power component of COT is decreased as walking velocity increased. At $1.38 \mathrm{~m} / \mathrm{s}$ of the walking speed, a occupancy of the electric power consumption and the gravitational support power consumption in the total power consumption is $17 \%$ whereas at $0.22 \mathrm{~m} / \mathrm{s}$ the occupancy is $39 \%$ As a result, the total COT is asymptotic to the mechanical component of COT. Therefore, we can confirm that the total COT is decreased as increase of walking velocity because of reducing the electric power consumption and the gravitational support power.

The mechanical component of COT is almost around 1.75 and it gradually decreased as increase of velocity. The reason of gentle decrease of mechanical COT is considered as the changing of the stride. By increasing the stride, the required number of steps to walk a desired distance is reduced. Hence, the number of acceleration and deceleration to rotate the leg unit is also decreased and causes decrease of mechanical energy consumption.

\section{Discussion}

\section{Comparison with other robots}

We compared minimum COT and maximum walking velocity of TITAN-XIII with other quadruped robots. Figure 21 shows the graph of minimum COT vs mass. Black markers indicate robots and white markers indicate animals which is adopted from Tucker's graph [20]. As we previously mentioned, in the case of a sprawlingtype quadruped robot, there is no research about energy efficiency except our previous robot TITAN-VIII [16]. 


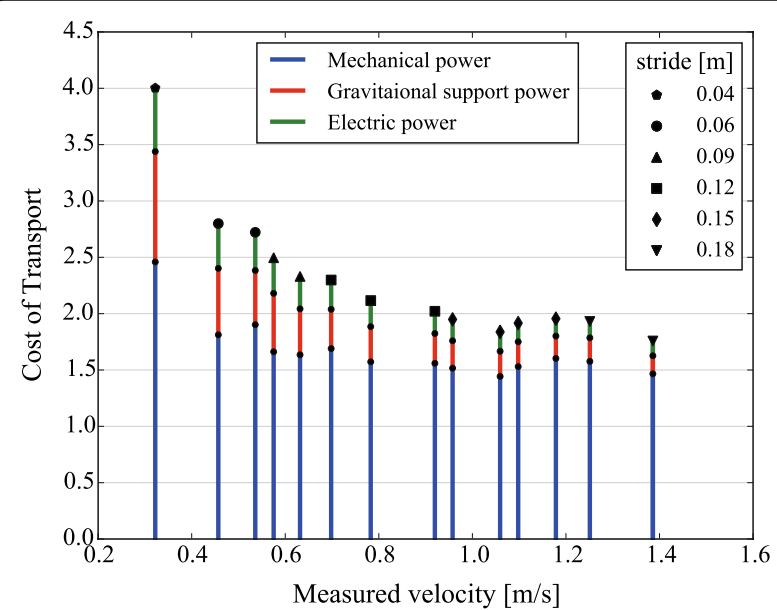

Fig. 20 COT vs velocity of TITAN-XIII: Each bar indicates total COT which include all energy consumption of the robot. Blue bar indicates the mechanical component of COT, red bar indicates gravitational support component of COT and green bar indicates electric component of COT. Net COT which exclude electric component is indicated as summation of blue bar and red bar

Therefore, most of the robots in the graph are a mammaltype quadruped robot.

Looking at other surrounding TITAN-XIII, the Scout II shows lower COT than TITAN-XIII, however Scout II can not select foot placement three dimensionally because of 4 DoF with hopping optimized leg design. Although StarlETH also shows slightly lower COT than TITAN-XIII, the weight of StarlETH is almost four times higher than TITAN-XIII. According to Tucker's graph, in the case of running animals minimum COT is proportional to the 0.3 power of the body weight, which is shown as a line in the Fig. 21. Therefore, a plot which is close or overcome this animal line means high performance and among the plotted robots, only MIT Cheetah overcomes this line.

Estimated minimum COT of the animal which has same weight with TITAN-XIII is around 1.0. This value is not so far from current lowest COT of 1.76. Actually except MIT Cheetah, TITAN-XIII is located closest to the estimated COT line of the animals.

We also plotted the lowest COT of 1.2 of TITAN-XIII with additional $5 \mathrm{~kg}$ of weight at $0.83 \mathrm{~m} / \mathrm{s}$ of the walking velocity in Fig. 21. Comparing COT of the robot without payload, the COT of the robot with payload is close to animal line. Therefore we can say TITAN-XIII is not just optimized for fast walking but possible to achieve payload and energy efficiency at a same time.

Next, we compare walking velocity of quadruped robot by using Froude number. Froude number represents a normalization of the walking velocity for the size of the robot, and higher Froude number means faster walking performance regardless. Froude number is defined as Froude number $=v^{2} / \mathrm{Lg}$, where the $v[\mathrm{~m} / \mathrm{s}]$ is walking velocity, the $L[m]$ is representative length and $g$ is the gravitational acceleration [21]. According to literature [22] by Alexander, representative length: $\mathrm{L}$ is defined as "the height of the hip joint from the ground in normal standing". However, using the height of the hip joint to calculate Froude number is not suit for a sprawling-type quadruped robot, because in case of a sprawling-type robot, the height of the hip joint can be decreased until its belly touches to the ground without decreasing possible stride. We have considered important length to calculate Froude number is the length which actually generate speed. Thus we have extended the Alexander's definition of representative length to "the length between the hip joint axis and the foot projected on a plane orthogonal to the moving direction in normal standing posture"?. By using this extended definition of representative length, Froude number can be calculated for both the mammaltype and the sprawling-type. Especially in case of mammal-type, if the foot is on the same sagittal plane of the hip joint, the extended definition will get same result as the Alexander's definition. According to the extended definition, representative length of TITAN-XIII in walking experiment is 0.172 as shown in Fig. 18.

Figure 22 shows the graph of Froude numbers vs mass of quadruped robots and animals. As shown in the graph, velocity of TITAN-XIII is almost same level as other mammal-type quadruped robots such as Cheetah-cub and Raibert quadruped and much faster than TITAN-VIII.

From the comparison of COT and Froude number, we can say that the walking speed and energy efficiency of the sprawling-type quadruped robot can be the same level as the mammal-type quadruped robot. Especially, the energy efficiency of the TITAN-XIII is closer to animal line than most of mammal-type quadruped robot.

\section{Validity of the sprawling-type quadruped robot}

As we mentioned in the previous section, TITAN-XIII can achieve high walking velocity and energy efficiency than previous sprawling-type quadruped robot and its performance is almost same level as a mammal-type quadruped robot. Additionally the payload of TITANXIII which is almost same as its own weight, is relatively better than other mammal-type quadruped robots. Considering the nature of the sprawling-type robot which is high static stability and wide range of motion of the foot, we believe the sprawling-type quadruped robot is highly practical.

However we also see trade-off problem about energy efficiency and rough terrain adaptability. Although 


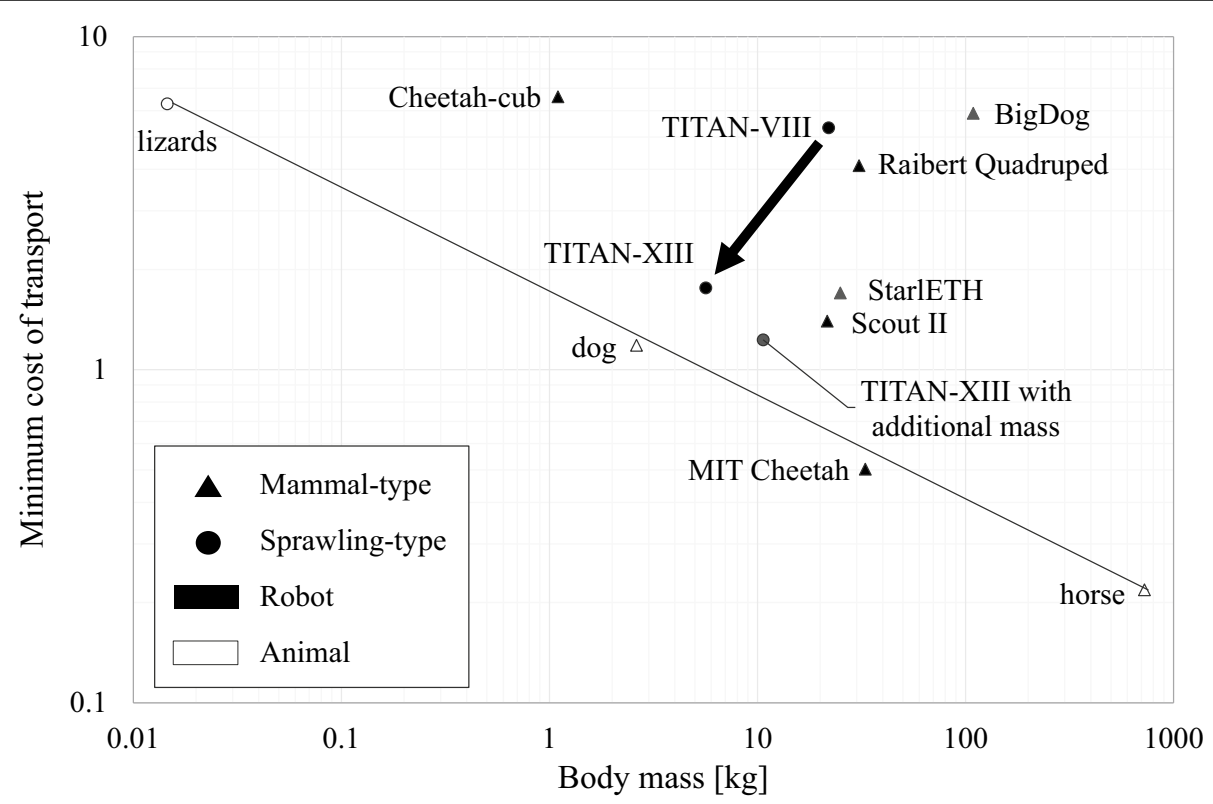

Fig. 21 Minimum Cost of Transport vs body mass of quadruped robots. This plot is based on Tucker's graph [20] and added relatively new quadruped robots $[3,5,16,24-27]$. The black line is drawn by the author

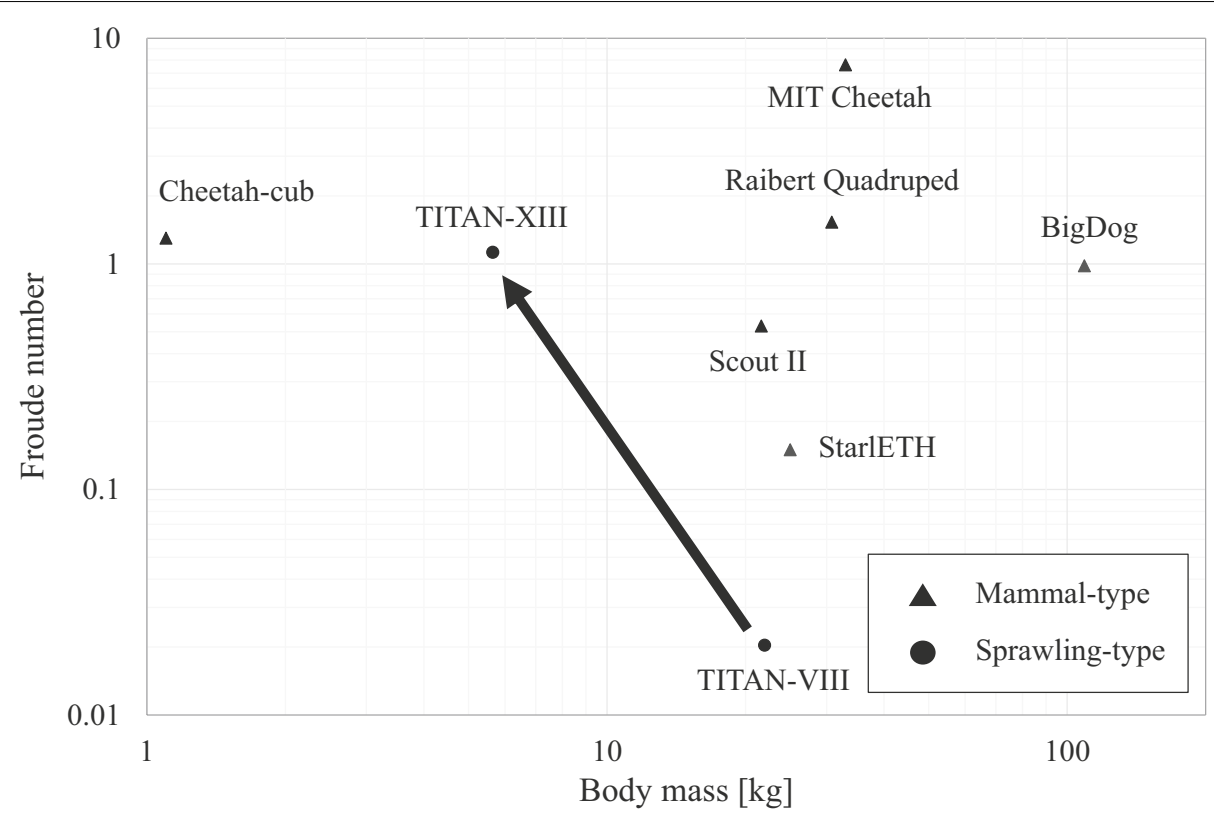

Fig. 22 Froude number vs mass of quadruped robots. Each data is based on Sprowits's data [5] and added by the author [3, 15]

energy efficiency and payload can be increased by increasing gear ratio of the actuator which supports gravitational force, at a same time vertical velocity of the foot is decreased and resulted low tracking performance in vertical direction as shown in Fig. 17. This low vertical velocity of the foot is problematic when the robot overcomes a obstacles, and eventually limit the maximum walking speed on rough terrain.

To solve this problem in a fundamental way, the changing reduction ratio depending on the walking phase is required as TITAN VI equipped [23]. However usually such kind of mechanism makes a robot heavy. Another 
solution is using the actuator of Axis 3 to generate the vertical velocity of the foot. Currently the Axis 3 joint does not move so much while walking because of parallel link mechanism. Therefore if we design another swing leg trajectory which is not in sagittal plane, the Axis 3 can be utilized and increase vertical velocity of the foot.

\section{Conclusion}

In this paper, to improve speed and energy efficiency of a sprawling-type quadruped robot, we focused on three design concepts (increase of walking velocity to reduce cost of transport, using the trot gait as low duty factor and safety gait, and increase of gear ratio of the actuator supporting gravitational force). Based on the concepts, we developed a sprawling-type quadruped robot named TITAN-XIII. To achieve a compact and low inertia leg, right-angle type wire driven mechanism was proposed and adopted to the robot. To confirm the validity of the developed sprawling-type quadruped robot, several experiments was conducted. In the experiment, the robot walked at $1.38 \mathrm{~m} / \mathrm{s}$ and minimum cost of transport of 1.76 was achieved. We compared the walking speed and minimum COT with other quadruped robots. In comparison, TITAN-XIII shows almost the same level of walking velocity and energy efficiency. Considering advantages of the sprawling-type quadruped robot, which are static stability and wide range of motion, we can say the sprawling-type quadruped robot is highly practical on rough terrain.

In the future work, we will conduct walking experiments with the developed robot on more difficult terrain and evaluate effectiveness of a sprawling-type quadruped robot.

\section{Authors' contributions}

All authors equally contributed. All authors read and approved the final manuscript.

\section{Author details}

${ }^{1}$ Department of Mechanical and Aerospace Engineering, Tokyo Institute of Technology, Meguro-ku, Ookayama 2-12-1, 152-8550 Tokyo, Japan. ${ }^{2}$ Hibot Corp., 5-9-15 Kitashinagawa, Shinagawa-ku, 141-0001 Tokyo, Japan.

\section{Acknowledgements}

This work was supported by Grant-in-Aid for Scientific Research (C) 25420214.

\section{Competing interests}

The authors declare that they have no competing interests.

Received: 28 November 2015 Accepted: 23 February 2016

Published online: 16 March 2016

\section{References}

1. Raibert M, Blankespoor K, Gabriel N, Playter R, the Big-Dog Team (2008) BigDog, the Rough-Terrain Quadruped Robot. In: Proceedings of the 17th World Congress. The International Federation of Automatic Control Seoul, Korea, July 6-11, 2008
2. Semini C, Tsagarakis NG, Guglielmino E, Focchi M, Cannella F, Caldwell DG (2011) Design of hyq, a hydraulically and electrically actuated quadruped robot. Proceedings of the Institution of Mechanical Engineers, Part I: Journal of Systems and Control Engineering 225(6):831-849. doi:10.1177/0959651811402275. URL: http://pii.sagepub.com/content/225/6/831.full.pdf+html

3. Seok S, Wang A, Chuah MYM, Hyun DJ, Lee J, Otten DM, Lang JH, Kim $S$ (2015) Design principles for energy-efficient legged locomotion and implementation on the mit cheetah robot. Mechatronics, IEEE/ASME Transactions on 20(3):1117-1129. doi:10.1109/TMECH.2014.2339013

4. Hutter M, Gehring C, Hopflinger MA, Blosch M, Siegwart R (2014) Toward combining speed, efficiency, versatility, and robustness in an autonomous quadruped. Robotics, IEEE Transactions on 30(6):1427-1440. doi:10.1109/TRO.2014.2360493

5. Sprowitz A, Tuleu A, Vespignani M, Ajallooeian M, Badri E, ljspeert AJ (2013) Towards dynamic trot gait locomotion: design, control, and experiments with Cheetah-cub, a compliant quadruped robot. Int J Robotics Res 32(8):932-950. doi:10.1177/0278364913489205

6. Fukuoka Y, Kimura H, Cohen AH (2003) Adaptive dynamic walking of a quadruped robot on irregular terrain based on biological concepts. Int J Robotics Res 22(3-4):187-202. doi: 10.1177/0278364903022003004. URL: http://ijr.sagepub.com/content/22/3-4/187.full.pdf+html

7. Kalakrishnan M, Buchli J, Pastor P, Mistry M, Schaal S (2011) Learning, planning, and control for quadruped locomotion over challenging terrain. Int J Robotics Res 30(2):236-258. doi:10.1177/0278364910388677

8. Zico Kolter J, Ng AY (2011) The stanford littledog: a learning and rapid replanning approach to quadruped locomotion. Int J Robotics Res 30(2):150-174. doi: 10.1177/0278364910390537. URL: http://ijr.sagepub. com/content/30/2/150.full.pdf+html

9. Neuhaus PD, Pratt JE, Johnson MJ (2011) Comprehensive summary of the institute for human and machine cognition's experience with littledog. Int J Robotics Res 30(2): 216-235. doi: 10.1177/0278364910390538. URL: http://ijr.sagepub.com/content/30/2/216.full.pdf+html

10. Hirose S, Fukuda Y, Kikuchi H (1986) The gait control system of a quadruped walking vehicle. Adv Robotics 1(4):289-323. doi:10.1163/1568553 $86 \times 00193$

11. Garcia E, de Santos PG (2006) On the improvement of walking performance in natural environments by a compliant adaptive gait. Robotics, IEEE Transactions on 22(6):1240-1253. doi:10.1109/TRO.2006.884343

12. Loc V-G, Koo I, Tran D, Park S, Moon H, Choi H (2012) Body workspace of quadruped walking robot and its applicability in legged locomotion. J Intell Robotic Sys 67(3-4):271-284. doi:10.1007/s10846-012-9670-0

13. Wait KW (2010) The use of pneumatic actuation to address shortcoming concerning normalized output power in state of the art mobile tobotics. $\mathrm{PhD}$ thesis, Graduate School of Vanderbilt University

14. Gabrielli G, von Karman T (1950) What price speed? Mechanical Eng 72(10):775-781

15. Arikawa K, Hirose S (Nov) Development of quadruped walking robot titan-viii. In: Intelligent Robots and Systems '96, IROS 96, Proceedings of the 1996 IEEE/RSJ International Conference On, vol 1, pp. 208-2141. doi:10.1109/IROS.1996.570670

16. Endo G, Hirose S (2012) Study on roller-walker-improvement of locomotive efficiency of quadruped robots by passive wheels. Adv Robotics 26:969-988. doi:10.1163/156855312X633066

17. TOYOBO dyneema basic properties. http://www.toyobo-global.com/ seihin/dn/dyneema/seihin/. Accessed Mar 2015

18. Kitano S, Hirose S, Endo G, Fukushima EF (2013) Development of lightweight sprawling-type quadruped robot titan-xiii and its dynamic walking. In: Intelligent robots and systems (IROS), 2013 IEEE/RSJ International Conference On, pp 6025-6030. doi:10.1109/IROS.2013.6697231

19. Hirose S, Tsukagoshi H, Yoneda K (2001) Normalized energy stability margin and its contour of walking vehicles on rough terrain. In: Robotics and Automation, 2001. Proceedings 2001 ICRA. IEEE International Conference On, vol 1, pp 181-1861. doi:10.1109/ROBOT.2001.932550

20. Tucker VA (1975) The energetic cost of moving about: walking and running are extremely inefficient forms of locomotion. much greater efficiency is achieved by birds, fish-and bicyclists. Am Sci 63(4):413-419

21. Alexander RM, Jayes AS (1983) A dynamic similarity hypothesis for the gaits of quadrupedal mammals. J Zool 201(1):135-152. doi:10.1111/j.1469-7998.1983.tb04266.x 
22. Alexander RM (2003) Principles of animal locomotion. Princeton University Press, Princeton, pp 58-60

23. Hirose S, Yoneda K, Arai K, Ibe T (1991) Design of prismatic quadruped walking vehicle titan vi. In: Advanced Robotics, 1991. 'Robots in Unstructured Environments', 91 ICAR., Fifth International Conference On, pp 723-7281. doi:10.1109/ICAR.1991.240685

24. Hutter M, Gehring C (2013) Walking and running with StarlETH. In: The 6th international symposium on adaptive motion of animals and machines (AMAM), pp 5-9. URL: http://publications.asl.ethz.ch/files/hutter13dynamic.pdf
25. Poulakakis I, Smith JA, Buehler M (2005) Modeling and experiments of untethered quadrupedal running with a bounding gait: The scout ii robot. Int J of Robotics Res 24(4):239-256. doi:10.1177/0278364904050917. URL: http://ijr.sagepub.com/content/24/4/239.full.pdf+html

26. BigDog overview[Online]. URL: http://www.bostondynamics.com/img/ BigDogOverview.pdf

27. Raibert MH, Brown H, Benjamin J, Chepponis M, Koechling J, Hodgins JK, Dustman D, Brennan WK, Barrett DS, Thompson CM, Hebert JD, Lee W, Borvansky L (1989) Dynamically stable legged locomotion. MIT Technical Report, 134

\section{Submit your manuscript to a SpringerOpen ${ }^{\circ}$ journal and benefit from:}

- Convenient online submission

- Rigorous peer review

- Immediate publication on acceptance

- Open access: articles freely available online

- High visibility within the field

- Retaining the copyright to your article

Submit your next manuscript at $>$ springeropen.com 